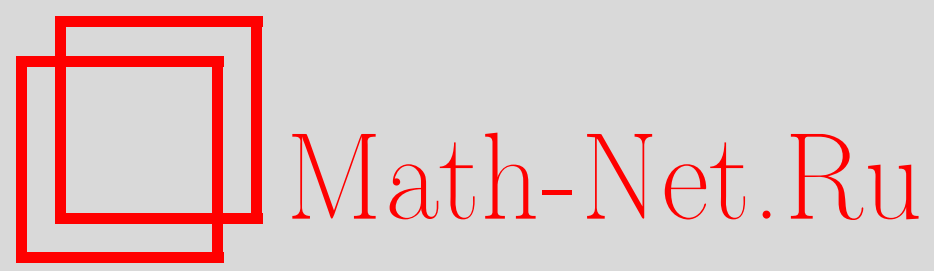

А. Ф. Заусаев, А. А. Заусаев, А. Г. Ольхин, Оценка точности метода Эверхарта при решении уравнений движения больших планет на интервале времени 10000 лет, Вестн. Сам. гос. техн. ун-та. Сер. Физ.-мат. науки, 2004, выпуск 30, 108 113

DOI: https://doi.org/10.14498/vsgtu315

Использование Общероссийского математического портала Math-Net.Ru подразумевает, что вы прочитали и согласны с пользовательским соглашением

http://www.mathnet.ru/rus/agreement

Параметры загрузки:

IP : 54.198 .187 .58

26 апреля 2023 г., 15:04:01 


\title{
Численные методы
}

\author{
УДК 521.1, 521.4
}

А. Ф. Заусаев, А. А. Заусаев, А. Г. Ольхин

\section{ОЦЕНКА ТОЧНОСТИ МЕТОДА ЭВЕРХАРТА ПРИ РЕШЕНИИ УРАВНЕНИЙ ДВИЖЕНИЯ БОЛЬШИХ ПЛАНЕТ НА ИНТЕРВАЛЕ ВРЕМЕНИ 10000 ЛЕТ}

Исследована возможность применения метода Эверхарта для решения уравнений движения планет на интервале времени 10000 лет. Оченка точности метода была проведена на основе алгоритма численного интегрирования 23 порядка с шагом 3 дня.

Целью данной работы является решение двух задач: а) исследование точности метода Эверхарта при интегрировании уравнений движения больших планет (Меркурий-Плутон) на интервале времени порядка 10000 лет, путем решения задачи $n$ тел; б) разработка аппроксимирующих формул высокого порядка.

Создаваемые ранее базы данных координат больших планет охватывали период телескопических наблюдений, начиная с 1660 г. [1]. Для исследования эволюции орбит малых тел Солнечной системы этот интервал времени мал, так как период изменения угловых элементов орбит составляет десятки и сотни тысяч лет.

Применяемые явные многошаговые методы Коуэлла не позволяли увеличить интервал интегрирования более чем на 500 лет, из-за быстрых накоплений ошибок округления [2]. Разработанный Эверхартом метод численного интегрирования обыкновенных дифференциальных уравнений относится к числу неявных одношаговых методов [3].

Основным достоинством одношаговых методов является то обстоятельство, что для них разработаны надежные оценки локальной погрешности дискретизации. Существуют три основных метода для оценки полной погрешности: экстраполяция, вложенные методы, многошаговые оценки погрешности [4]. Экстраполяция и вложенные методы являются наиболее распространенными для оценок полной погрешности метода интегрирования. Экстраполяция основана на следующей идее: интегрирование производится дважды, с шагом $h$ и $h / 2$. Тогда погрешность при шаге $h / 2$ приблизительно равна разности решений с шагом $h$ и $h / 2$, умноженной на $2^{-p}[4]$.

Для оценки погрешности вложенным методом, интегрирование также выполняется дважды и по разности полученных решений дается оценка полной погрешности на конце интервала интегрирования.

Известно, что точность метода численного интегрирования обыкновенных дифференциальных уравнений можно повысить двумя способами: путем уменьшения длины шага интегрирования либо увеличением порядка аппроксимирующей формулы. Уменьшение шага интегрирования на больших интервалах времени может привести к увеличению ошибки округления. Увеличение порядка аппроксимирующей формулы при условии, что задача Коши не является жесткой, и производные высших порядков не претерпевают разрывов, увеличивает точность метода при постоянном шаге. Кроме того, для алгоритмов Рунге-Кутты область абсолютной устойчивости метода увеличивается с увеличением его порядка [5].

Вследствие того, что повышение порядка аппроксимирующей формулы, в большинстве случаев, улучшает основные свойства методов, разработка методов Эверхарта более высокого порядка является актуальной задачей не только с целью исследования полной погрешности вложенных методов, но и с точки зрения создания более эффективного алгоритма численного интегрирования. С этой целью нами разработаны алгоритмы для метода Эверхарта до 33 порядка включительно. 
1. Метод Эверхарта. Метод Эверхарта является одной из разновидностей методов РунгеКутты. Он относится к неявным одношаговым методам, что обеспечивает его сходимость и устойчивость $[3,6]$. Рассмотрим основную идею построения метода Эверхарта на примере решения уравнения вида

$$
\ddot{x}=F(x, t) .
$$

Представим правую часть (1) в виде временного ряда

$$
\ddot{x}=F(x, t)=F_{1}+A_{1} t+A_{2} t^{2}+\ldots+A_{n} t^{n} .
$$

Интегрируя (2), получим выражения для определения координат и скоростей:

$$
\begin{gathered}
x=x_{1}+\dot{x}_{1} t+F_{1} \frac{t^{2}}{2}+A_{1} \frac{t^{3}}{6}+\ldots+A_{n} \frac{t^{n+2}}{(n+1)(n+2)}, \\
\dot{x}=\dot{x}_{1}+F_{1} t+A_{1} \frac{t^{2}}{2}+A_{2} \frac{t^{3}}{6}+\ldots+A_{n} \frac{t^{n+1}}{n+1} .
\end{gathered}
$$

Полиномы (3) и (4) не являются рядами Тейлора, а коэффициенты $A_{i}$ вычисляются из условия наилучшего приближения $x$ и $\dot{x}$ с помощью конечных разложений (3) и (4). Для связи $A$ значений с $F$-значениями воспользуемся вспомогательным выражением

$$
F=F_{1}+\alpha_{1} t+\alpha_{2} t\left(t-t_{2}\right)+\alpha_{3} t\left(t-t_{2}\right)\left(t-t_{3}\right)+\ldots
$$

Уравнение (5) усечено по времени $t_{n}$. В каждый фиксированный момент времени $t_{i}$ имеем

$$
\begin{aligned}
& F_{2}=F_{1}+\alpha_{1} t_{2}, \\
& F_{3}=F_{1}+\alpha_{1} t_{3}+\alpha_{2} t_{3}\left(t_{3}-t_{2}\right),
\end{aligned}
$$

Принимая $t_{n j}=t_{n}-t_{j}$, найдем $\alpha_{i}$ через разделенные разности:

$$
\begin{aligned}
& \alpha_{1}=\left(F_{2}-F_{1}\right) / t_{2}, \\
& \alpha_{2}=\left(\left(F_{3}-F_{1}\right) / t_{3}-\alpha_{1}\right) / t_{32}, \\
& \alpha_{3}=\left(\left(\left(F_{4}-F_{1}\right) / t_{4}-\alpha_{1}\right) / t_{42}-\alpha_{2}\right) / t_{43}, \\
& \alpha_{4}=\left(\left(\left(\left(F_{5}-F_{1}\right) / t_{5}-\alpha_{1}\right) / t_{52}-\alpha_{2}\right) / t_{53}-\alpha_{3}\right) / t_{54},
\end{aligned}
$$

Приравнивая коэффициенты при одинаковых степенях $t$ в уравнениях (2) и (5), выразим коэффициенты $A_{i}$ через $\alpha_{i}$ :

$$
\begin{aligned}
& A_{1}=\alpha_{1}+\left(-t_{2}\right) \alpha_{2}+\left(t_{2} t_{3}\right) \alpha_{3}+\ldots=c_{11} \alpha_{1}+c_{21} \alpha_{2}+c_{31} \alpha_{3}+\ldots, \\
& A_{2}=\alpha_{2}+\left(-t_{2}-t_{3}\right) \alpha_{3}+\ldots=c_{22} \alpha_{2}+c_{32} \alpha_{3}+\ldots, \\
& A_{3}=\alpha_{3}+\ldots=c_{33} \alpha_{3}+\ldots
\end{aligned}
$$

Коэффициенты $c_{i j}$ определяются из следующих рекуррентных соотношений:

$$
\begin{aligned}
& c_{i j}=1, \quad i=j, \\
& c_{i 1}=-t_{i} c_{i-1,1}, \quad i>1, \\
& c_{i j}=c_{i-1, j-1}-t_{i} c_{i-1, j} \quad 1<j<i .
\end{aligned}
$$

Для алгоритма интегрирования пятого порядка имеем:

$$
c_{41}=-t_{2} t_{3} t_{4}, c_{42}=t_{2} t_{3}+t_{3} t_{4}, c_{43}=-t_{2}-t_{3}-t_{4},
$$

где $t_{2}, t_{3}, t_{4}$ являются корнями кубического уравнения

$$
\left(-t_{2} t_{3} t_{4}\right)+\left(t_{2} t_{3}+t_{3} t_{4}+t_{4} t_{2}\right) t+\left(-t_{2}-t_{3}-t_{4}\right) t^{2}+(1) t^{3}=0
$$

Таким образом, нахождение решения уравнения (1) сводится к нахождению узлов разбиения $t_{i}$ шага $h$.

2. Нахождение узлов разбиения шага интегрирования. Нахождение узлов разбиения шага $h=[0, T]$ рассмотрим на примере алгоритма интегрирования пятого порядка. 
В начальный момент времени $t_{1}=0$ известны $x_{1}, \dot{x}_{1}, F_{1}$. Значения $x$ в моменты времени $t_{2}, t_{3}, t_{4}$ определяются с помощью трех предсказывающих уравнений:

$$
\begin{aligned}
& x_{2}=x_{1}+\dot{x}_{1} t_{2}+F_{1} t_{2}^{2} / 2+\left[A_{1} t_{2}^{3} / 6+A_{2} t_{2}^{4} / 12+A_{3} t_{2}^{5} / 20\right] ; \\
& x_{3}=x_{1}+\dot{x}_{1} t_{3}+F_{1} t_{3}^{2} / 2+A_{1} t_{3}^{3} / 6+\left[A_{2} t_{3}^{4} / 12+A_{3} t_{3}^{5} / 20\right] ; \\
& x_{4}=x_{1}+\dot{x}_{1} t_{4}+F_{1} t_{4}^{2} / 2+A_{1} t_{4}^{3} / 6+A_{2} t_{4}^{4} / 12+\left[A_{3} t_{4}^{5} / 20\right] ;
\end{aligned}
$$

и двух исправляющих уравнений для нахождения положения и скорости на конце шага $h$ :

$$
\begin{gathered}
x(T)=x_{1}+\dot{x}_{1} T+F_{1} T^{2} / 2+A_{1} T^{3} / 6+A_{2} T^{4} / 12+A_{3} T^{5} / 20 ; \\
\dot{x}(T)=\dot{x}_{1}+F_{1} T+A_{1} T^{2} / 2+A_{2} T^{3} / 3+A_{3} T^{4} / 4 .
\end{gathered}
$$

Эта схема является неявной, так как коэффициенты, стоящие в квадратных скобках (12-14), неизвестны при первой итерации.

Уравнения (12)-(16) обеспечивают пятый порядок точности относительно $t$. Можно увеличить порядок точности в вычислении $x$ и $\dot{x}$ до седьмого порядка путем специального выбора подшагов $t_{2}, t_{3}, t_{4}$. С этой целью увеличим количество разбиений интервала интегрирования, добавив два дополнительных времени $t_{5}, t_{6}$. Затем вычислим для $t_{5}$ и $t_{6}$ значения $\alpha_{4}$ и $\alpha_{5}$, a также новые значения $A_{4}^{\prime}, A_{5}^{\prime}$ и $A_{1}^{\prime}, A_{2}^{\prime}, A_{3}^{\prime}$.

Из уравнения (15) можно найти поправки $\Delta x$, улучшающие значения координат:

$$
\Delta x=\left(A_{1}^{\prime}-A_{1}\right) T^{3} / 6+\left(A_{2}^{\prime}-A_{2}\right) T^{4} / 12+\left(A_{3}^{\prime}-A_{3}\right) T^{5} / 20+A_{4}^{\prime} T^{6} / 30+A_{5}^{\prime} T^{7} / 42 .
$$

Выражая в уравнении (9) $c_{51}, \ldots, c_{54}$ через $c_{41}, c_{42}, c_{43}$, а также полагая

$$
h_{2}=t_{2} / T, h_{3}=t_{3} / T \text { и } h_{4}=t_{4} / T,
$$

формула (17) может быть записана в виде

$$
\Delta x=\left(\alpha_{4}-t_{5} \alpha_{5}\right) T^{6}\left[c_{41}^{\prime} / 6+c_{42}^{\prime} / 12+c_{43}^{\prime} / 20+1 / 30\right]+\alpha_{5} T^{7}\left[c_{41}^{\prime} / 12+c_{42}^{\prime} / 30+1 / 42\right] .
$$

Значение $\Delta x$ в последнем выражении можно обратить в ноль при выполнении следующих условий:

$$
\begin{aligned}
& c_{41}^{\prime} / 6+c_{42}^{\prime} / 12+c_{43}^{\prime} / 20+1 / 30=0, \\
& c_{41}^{\prime} / 12+c_{42}^{\prime} / 20+c_{43}^{\prime} / 30+1 / 42=0 .
\end{aligned}
$$

Проводя подобные рассуждения для скорости, приравнивая к нулю $\Delta \dot{x}$, получим третье условие для определения $c_{41}^{\prime}, c_{42}^{\prime}, c_{43}^{\prime}$. Тогда соответствующие данным разбиениям коэффициенты $c_{i j}^{\prime}$ будут определяться из системы алгебраических уравнений вида

$$
\left\{\begin{array}{l}
c_{41}^{\prime} / 2+c_{42}^{\prime} / 3+c_{43}^{\prime} / 4+1 / 5=0, \\
c_{41}^{\prime} / 3+c_{42}^{\prime} / 4+c_{43}^{\prime} / 5+1 / 6=0, \\
c_{41}^{\prime} / 4+c_{42}^{\prime} / 5+c_{43}^{\prime} / 6+1 / 7=0 .
\end{array}\right.
$$

Из решения этой системы

$$
c_{41}^{\prime}=-4 / 35=-h_{2} h_{3} h_{4} ; c_{42}^{\prime}=6 / 7=h_{2} h_{3}+h_{3} h_{4}+h_{2} h_{4} ; c_{43}^{\prime}=-12 / 7=-h_{2}-h_{3}-h_{4}
$$

следует, что значения величин $h_{2}, h_{3}, h_{4}$ являются корнями следующего полинома третьей степени:

$$
h^{3}+(-12 / 7) h^{2}+(6 / 7) h-4 / 35=0 .
$$

которые имеют следующие значения:

$$
\begin{gathered}
h_{2}=t_{2} / T=0.212340538239 \ldots ; h_{3}=t_{3} / T=0.590533135559 \ldots ; \\
h_{4}=t_{4} / T=0.91141240488 \ldots
\end{gathered}
$$

Использование этих узлов позволяет получить решение уравнения (1) с точностью до седьмого порядка для обеих компонент $x$ и $\dot{x}$. Полученные по формуле (22) узлы разбиения $h$ совпадают с узлами квадратурной формулы Гаусса-Радо. Область изменения $h$ заключена в пределах $0 \leq h \leq 1$.

Таким образом, порядок метода, определяющий точность интегрирования, зависит от количества разбиений основного шага $h$ на подшаги $h_{i}$. 
На данный метод Э. Эверхартом разработаны алгоритмы и программа на языке Фортран. Программа Эверхарта адаптирована на ЭВМ БЭСМ-6 С.В. Тарасевием в Институте теоретической астрономии АН СССР.

В работе Э. Эверхарта [3] приведены узлы разбиения основного шага интегрирования на подшаги, обеспечивающие точность до 15 порядка включительно.

В табл. 1 приведены узлы разбиения отрезка $[0,1]$ на подшаги $h_{i}=\frac{t_{i}}{T}$ для 19 и 23 порядков метода.

Известно, что положение небесного объекта в заданной системе координат определяется шестью элементами орбит: $M, a, e, \omega, \Omega, i$, где $M$ - средняя аномалия, $a$-большая полуось, $e$ эксцентриситет, $\omega$-аргумент перигелия, $\Omega$-долгота восходящего узла, $i$-наклонение.

Положение планеты на орбите определяется двумя величинами: радиус-вектором $r$ и истинной аномалией $V$. Средняя аномалия представляет собой дугу круга, которую описывала бы планета за время $\left(t-t_{0}\right)$, если бы она двигалась равномерно по окружности радиуса $a$ со средней угловой скоростью $n$. Связь средней аномалии с эксцентрической определяется уравнением Кеплера

$$
E-e \sin E=n(t-T)=M .
$$

Истинная аномалия выражается через эксцентрическую с помощью формулы

$$
\operatorname{tg} \frac{V}{2}=\sqrt{\frac{1+e}{1-e}} \operatorname{tg} \frac{E}{2} .
$$

Таким образом, зная $M$ - среднюю аномалию, можно легко определить $V$ - истинную аномалию.

Для оценки интервала интегрирования, на котором можно эффективно использовать метод Эверхарта для получения координат больших планет, нами проведено численное интегрирование уравнений движения больших планет [2] с шагом 0.5 дня и 1, 2, 3 дня с различным порядком точности, 19 и 23 соответственно.

Начальные данные масс, координат и скоростей планет (Меркурий-Плутон) на момент времени $2433280.5 \mathrm{JED}$ взяты из Справочного руководства по небесной механике и астродинамике [7].

В настоящее время имеются более точные начальные данные координат и масс больших планет. В данной работе не ставилось целью получить на конце интервала интегрирования координаты и скорости планет с максимально возможной точностью. Целью исследования являлось сопоставление результатов вычислений при использовании метода с различным шагом и порядком, поэтому выбор начальных данных не может повлиять на основные выводы.

Численные результаты элементов орбит планет, полученные методом Эверхарта с различным шагом интегрирования и различным порядком, приведены в табл. 2, 3 .

Данные эксперимента показывают, что на момент 2798530.5 JED результаты вычислений, полученные методом Эверхарта 19 и 23 порядками точности и шагом интегрирования 1 и 3 дня (соответственно) совпадают с точностью до шестого знака после запятой для всех элементов орбит, включая среднюю аномалию.

В табл. 3 приведены значения максимальной разности погрешности средней аномалии Меркурия на конце интервала интегрирования для различных вариантов метода Эверхарта.

Из сопоставления данных, приведенных в табл. 3, следует, что имеется незначительное расхождение в средней аномалии Меркурия, не превышающее 0.1 секунды дуги.

Оценка полной погрешности метода Эверхарта экстраполяционным и вложенным методами позволяет сделать следующий вывод: максимальная погрешность в средних аномалиях планет не превосходит 0.1 угловых секунд дуги.

Полученный результат позволяет применять метод Эверхарта с порядком не менее 19 и с шагом интегрирования не более 1 дня для создания банка данных координат больших планет (Меркурий-Плутон) на интервале времени порядка 10000 лет.

Основными вопросами при создании банка данных являются вопросы эффективности, точности и устойчивости используемых методов.

Повышение точности метода можно осуществлять двумя способами: путем увеличения порядка аппроксимирующей формулы или путем уменьшения шага интегрирования. К решению этой задачи необходимо относиться с большой осторожностью, так как значительное повышение порядка может привести к потере точности, вследствие того, что производные высо- 
кого порядка, вычисленные с помощью разностной схемы, теряют всякий физический смысл. С другой стороны, уменьшение шага вдвое, как правило, удваивает время вычислений, в то время как повышение порядка метода увеличивает работу в 1.3 раза. Сопоставляя эффективность различных модификаций метода Эверхарта, следует отметить, что метод Эверхарта с использованием 23 порядка и шагом интегрирования 3 дня является более эффективным, чем метод Эверхарта 19 порядка с шагом 1 день.

Как показывают расчеты, наибольшая погрешность наблюдается в средней аномалии Меркурия. Это обстоятельство накладывает ограничение на интервал времени, на котором координаты Меркурия могут быть вычислены точно. Для остальных планет (Венера-Плутон) точные координаты с ошибкой в средней аномалии менее 1 секунды дуги могут быть вычислены методом Эверхарта на значительно большем интервале времени, чем 10000 лет.

В связи с тем, что основной вклад в эволюционные процессы малых тел Солнечной системы вносят внешние планеты: Юпитер, Сатурн, Уран, Нептун, их координаты с помощью рассмотренных алгоритмов и программ можно вычислять с высокой степенью точности ( $\Delta M \leq 1^{\prime \prime}$ ) на значительно больших интервалах времени.

Разбиение интервала $(0,1)$ на подшаги Гаусса-Радо

Т а б л и ц а 1

\begin{tabular}{|c|c|}
\hline Порядок & $h_{n}$ \\
\hline 19 & 0.03625781288320946094 \\
& 0.11807897878999870019 \\
& 0.23717698481496038531 \\
& 0.38188276530470597536 \\
& 0.53802959891898906511 \\
& 0.69033242007236218294 \\
& 0.82388334383700471814 \\
& 0.92561261029080395536 \\
& 0.98558759035112345137 \\
\hline & 0.025273620397520349419925 \\
& 0.083041613447405145741918 \\
& 0.169175100377181424343219 \\
& 0.277796715109032072344951 \\
& 0.401502720232860814519170 \\
& 0.531862386910415955804065 \\
& 0.659991842085334810022770 \\
& 0.777159392956162143241701 \\
& 0.875380774855556925520646 \\
& 0.947964548872819447093136 \\
& 0.989981719538319594093396 \\
\hline
\end{tabular}

Т а б л и ц а 2

Элементы орбит планет (Меркурий-Плутон) 2950 г. T=2798530.5 JED (Метод Эверхарта 23-го порядка, шаг 1 день)

\begin{tabular}{|l|c|c|c|c|c|c|}
\hline \multicolumn{1}{|c|}{ Планеты } & $M$ & $a$ & $e$ & $\Omega$ & $\omega$ & $i$ \\
\hline Меркурий & 315.660663 & 0.387098 & 0.205833 & 31.668529 & 46.476440 & 6.944348 \\
Венера & 126.530241 & 0.723336 & 0.006357 & 57.364557 & 73.434882 & 3.382386 \\
Земля + Луна & 346.539534 & 1.000002 & 0.016276 & 293.225930 & 172.025772 & 0.129775 \\
Марс & 46.953004 & 1.523734 & 0.094188 & 293.406555 & 46.166782 & 1.766314 \\
Юпитер & 48.576655 & 5.207484 & 0.050057 & 274.755700 & 101.794149 & 1.290321 \\
Сатурн & 40.838023 & 9.568937 & 0.054025 & 347.718653 & 110.630736 & 2.508759 \\
Уран & 256.544033 & 19.299182 & 0.047036 & 93.079095 & 74.506645 & 0.758859 \\
Нептун & 168.376980 & 29.990290 & 0.012681 & 279.348638 & 131.213339 & 1.776997 \\
Плутон & 314.320135 & 39.614951 & 0.248680 & 113.123774 & 109.455772 & 17.119466 \\
\hline
\end{tabular}


Элементы орбит планет (Меркурий-Плутон) 11950 г. T=6085780.5 JED

Окончание табл. 2 (Метод Эверхарта 23-го порядка, иаг 1 день)

\begin{tabular}{|l|c|c|c|c|c|c|}
\hline \multicolumn{1}{|c|}{ Планеты } & $M$ & $a$ & $e$ & $\Omega$ & $\omega$ & $i$ \\
\hline Меркурий & 3.503077 & 0.387099 & 0.207237 & 57.051115 & 34.190516 & 6.451584 \\
Венера & 308.947234 & 0.723330 & 0.003504 & 65.142485 & 46.709965 & 3.001259 \\
Земля + Луна & 258.963782 & 1.000001 & 0.011470 & 345.277957 & 150.643196 & 1.180695 \\
Марс & 35.348060 & 1.523668 & 0.101270 & 12.288292 & 5.864932 & 0.804450 \\
Юпитер & 288.251259 & 5.208511 & 0.059721 & 280.864080 & 116.962633 & 1.444807 \\
Сатурн & 153.316517 & 9.589330 & 0.015922 & 90.326583 & 87.260732 & 2.297616 \\
Уран & 280.651233 & 19.141697 & 0.043851 & 100.926398 & 84.783361 & 0.648967 \\
Нептун & 44.428658 & 30.030115 & 0.010463 & 267.792744 & 130.507739 & 1.799445 \\
Плутон & 63.003967 & 39.623867 & 0.246783 & 115.648776 & 108.705552 & 17.128889 \\
\hline
\end{tabular}

Т а б л и ц а 3

Разности в средних аномалиях для Меркурия в различных вариантах метода Эверхарта. $T=6085780.5 \mathrm{JED}$

\begin{tabular}{|c|c|c|c|}
\hline$\Delta M\left(23^{n} 2^{d}\right.$ и $\left.23^{n} 1^{d}\right)$ & $\Delta M\left(19^{n} 0.5^{d}\right.$ и $\left.23^{n} 1^{d}\right)$ & $\Delta M\left(19^{n} 1^{d}\right.$ и $\left.23^{n} 1^{d}\right)$ & $\Delta M\left(23^{n} 3^{d}\right.$ и $\left.23^{n} 1^{d}\right)$ \\
\hline $0 . " 0612$ & $0 . .^{\prime \prime} 036$ & $0 . " 018$ & $0 . " 0648$ \\
\hline
\end{tabular}

БИБЛИОГРАФИЧЕСКИЙ СПИСОК.

1. Беляев Н.А. Эволюция орбиты кометы Даниэля 1909IV за 400 лет (1660-2060 гг.). Предварительное исследование // Л.: Бюлл.ИТА. 1966. Т.10. № 10(157). С.696-710.

2. Чеботарев Г.А. Аналитические и численные методы небесной механики. М.-Л.: Наука, 1965. 368 с.

3. Everhart E. Implicit sing1e methods for integrating orbits. // Ce1 estia1 mechanics. 1974. №.10. P.35-55.

4. Холл Д., Уатт Д.. Современные численные методы решения обыкновенных дифференциальных уравнений. М.: Мир, 1979. 312 с.

5. Бордовицина Т.В. Современные численные методы в задачах небесной механики. М.: Наука, 1984. 136 с.

6. Борунов В.П., Иванов В.А., Миронов С.В. Сравнение эффективности методов Фелберга, Дормана-Принса и Эверхарта численного интегрирования систем обыкновенных дифференциальных уравнений. Применение систем Mathematica и Maple в научных исследованиях. М.: ВЦ РАН, 2001, С.17-62.

7. Абалакин В.К., Аксенов Е.П., Гребенников Е.А., Рябов Ю.А. Справочное руководство по небесной механике. М.: Наука, 1976. 862 с. 